\title{
Inferring soil salinity in a drip irrigation system from multi-configuration EMI measurements using adaptive Markov chain Monte Carlo
}

\author{
Khan Zaib Jadoon ${ }^{1, a}$, Muhammad Umer Altaf ${ }^{2,3}$, Matthew Francis McCabe ${ }^{2}$, Ibrahim Hoteit ${ }^{3}$, Nisar Muhammad ${ }^{1}$, \\ Davood Moghadas ${ }^{4}$, and Lutz Weihermüller ${ }^{5}$ \\ ${ }^{1}$ Department of the Civil Engineering, COMSATS Institute of Information Technology, Abbottabad 22060, Pakistan \\ ${ }^{2}$ Water Desalination and Reuse Center, King Abdullah University of Science and Technology (KAUST), \\ Thuwal, 23955-6900, Saudi Arabia \\ ${ }^{3}$ Earth Science and Engineering, King Abdullah University of Science and Technology (KAUST), \\ Thuwal, 23955-6900, Saudi Arabia \\ ${ }^{4}$ Brandenburg University of Technology, Research Center Landscape Development and Mining Landscapes, \\ 03046 Cottbus, Germany \\ ${ }^{5}$ Agrosphere (IBG-3), Institute of Bio- and Geosciences, Forschungszentrum Jülich, GmbH, 52425 Jülich, Germany \\ ${ }^{a}$ now at: Department of Civil Engineering, International Islamic University, Islamabad 44000, Pakistan
}

Correspondence to: Khan Zaib Jadoon (khanzaib.jadoon@iiu.edu.pk)

Received: 12 June 2016 - Discussion started: 8 August 2016

Revised: 16 July 2017 - Accepted: 23 July 2017 - Published: 26 October 2017

\begin{abstract}
A substantial interpretation of electromagnetic induction (EMI) measurements requires quantifying optimal model parameters and uncertainty of a nonlinear inverse problem. For this purpose, an adaptive Bayesian Markov chain Monte Carlo (MCMC) algorithm is used to assess multi-orientation and multi-offset EMI measurements in an agriculture field with non-saline and saline soil. In MCMC the posterior distribution is computed using Bayes' rule. The electromagnetic forward model based on the full solution of Maxwell's equations was used to simulate the apparent electrical conductivity measured with the configurations of EMI instrument, the CMD Mini-Explorer. Uncertainty in the parameters for the three-layered earth model are investigated by using synthetic data. Our results show that in the scenario of non-saline soil, the parameters of layer thickness as compared to layers electrical conductivity are not very informative and are therefore difficult to resolve. Application of the proposed MCMC-based inversion to field measurements in a drip irrigation system demonstrates that the parameters of the model can be well estimated for the saline soil as compared to the non-saline soil, and provides useful insight about parameter uncertainty for the assessment of the model outputs.
\end{abstract}

\section{Introduction}

Electromagnetic induction (EMI) with low frequency is a powerful tool to map the hydrological processes in the vadose zone due to the sensitivity to water content and soil salinity (Robinson et al., 2009). The use of EMI is largely motivated by the need for robust and compact system design, ease of use, rapid acquisition, and capability to provide a large set of georeferenced measurements, which can be associated with the spatial variability of subsurface at the field scale (Corwin, 2008). The EMI instrument is used to measure soil apparent electrical conductivity $\left(\mathrm{EC}_{\mathrm{a}}\right)$, providing distribution of averaged electrical conductivity over a particular depth range. The depth of investigation of $\mathrm{EC}_{\mathrm{a}}$ depends on the coil spacing, the coil orientation, and the frequency of the energizing field. Mester et al. (2011) reported that in the low induction number condition, the coil orientation, offset, and frequency have major, moderate and minor effects on the penetration depth, respectively. Soil moisture, salinity, and texture cannot be directly observed with EMI measurements. However, in non-saline soils, cation exchange capacity, and soil moisture and texture are factors responsible for $\mathrm{EC}_{\mathrm{a}}$ variations (Rhoades et al., 1976; Sudduth et al., 2003), whereas 
in saline soil, the $\mathrm{EC}_{\mathrm{a}}$ measurement is generally dominated by the soil salinity, and the reason is the accumulation of more salt concentration in the topsoil due to the loss of water through evaporation (Corwin and Lesch, 2005a, b; Ershadi et al., 2014). The success of EMI measurements to assess soil salinity depends on the establishment of site-specific petrophysical relationships to relate $\mathrm{EC}_{\mathrm{a}}$ with the soil salinity estimated by electrical conductivity of the saturated paste extract $\left(\mathrm{EC}_{\mathrm{e}}\right)$ (Cook and Walker, 1992).

Several inversion algorithms have been developed for EMI measurements to improve the resolution of subsurface features and the assessment of soil properties (Hendrickx et al., 2002; Santos et al., 2010; Triantafilis and Monteiro Santos, 2013). The majority of these inversion algorithms solve a 1$\mathrm{D}$ earth model for electromagnetic wave propagation. The model of McNeill (1980) has been extensively used for low induction number and Maxwell's equations have been utilized for high-conductive soil $\left(\mathrm{EC}_{\mathrm{a}}>100 \mathrm{mS} \mathrm{m}^{-1}\right)$ where the low induction number assumption is not valid. For example, Li et al. (2013) used Geonics EM38 to measure EC $_{\mathrm{a}}$ in a rice paddy and inverted these using the McNeill (1980) forward model to estimate the variation of soil salinity in a field condition. They reported that the yield reduced by $33 \%$ in an irregularly shaped patch of strong saline topsoil.

EMI systems are sensitive to the field-specific calibration procedure, which limits the accuracy of $\mathrm{EC}_{\mathrm{a}}$ measurements. In inversion modeling, however, precise measurement of $\mathrm{EC}_{\mathrm{a}}$ is a prerequisite to characterize subsurface soil properties. For decades, the development and use of quantitative EMI inversions were mainly hampered by the lack of efficient calibration methods; von Hebel et al. (2014) used electrical resistivity tomography to calibrate EMI measurements before inversion to estimate three-dimensional images of subsurface electrical conductivity. Recently, Jadoon et al. (2015) calibrated EMI measurements via vertical electrical conductivity profile measured by capacitance sensors in different pits and later performed inversion for calibrated multi-configuration EMI measurements to estimate the effect of soil salinity distribution in an acacia tree farm.

EMI inversion algorithms are generally robust and provide useful estimates of subsurface properties in terms of optimal model parameters. Analysis of uncertainty in model parameters is however often left unaddressed. Parameter uncertainty can be associated with measurement errors (acquisition geometry, instrumental calibration and human error), modeling errors (assumptions in the electromagnetic forward model and petrophysical relationships), prior assumptions or constraints, parametrization, and estimation methods. Parameter uncertainty analysis can serve two main purposes: to identify the model parameters of dominant importance, and to provide confidence in the estimated model parameters (Scharnagl et al., 2011). For instance, Minsley (2011) used synthetic data considering the characteristics of the shallow ground-based EMI system, geophex GEM-2 (Huang and Won, 2003), to quantify the parameter uncertainty of a three-layer model via a Bayesian Markov chain Monte Carlo (MCMC) approach. They showed that combining multiple configuration EMI measurements significantly reduced total error, was best able to capture the shallow interface, and reduced regions of uncertainty at depth.

Conventional estimation of a single best-fit model with linear uncertainty does not usually trace ambiguity in the models, and may lead to a misguiding or imprecise interpretation. In this work, an adaptive Bayesian MCMC algorithm was used for inverting multi-orientation and multi-offset EMI measurements, in which the parameter posterior distribution represents the complete solution of the Bayesian inversion problem, including prediction of optimal parameter values and the associated uncertainty. Synthetic scenarios are first analyzed for a three-layered earth model to evaluate the uncertainty in model parameters for saline and non-saline soil using the characteristics of the CMD Mini-Explorer EMI system. Field measurements of the CMD Mini-Explorer are then used to quantify parameter uncertainties in the three-layered earth model and soil salinity distributions in an agricultural field irrigated with drip irrigation system.

\section{Materials and methods}

\subsection{Electromagnetic forward model}

Forward EMI response for a given layered earth model is usually calculated by the McNeill (1980) model, which is generated using the cumulative electrical conductivity distribution over a certain depth range, and is valid under condition of low induction number. The alternative method used to calculate the forward EMI response is to solve the Maxwell equation for the magnetic field measured over a horizontal layered medium (Keller and Frischknecht, 1966; Anderson, 1979). Preliminary analysis indicated that the electromagnetic forward model, which is based on high induction number assumption, returned more reliable apparent electrical conductivity values than the standard sensitivity curves of McNeill (1980). Furthermore, increased computational power made it possible to characterize the subsurface by utilizing forward models based on the Maxwell equation (Santos et al., 2010). The effective depth of exploration is independent of $\mathrm{EC}_{\mathrm{a}}$ in a low induction number condition, whereas in high induction number condition an inverse relationship was found between the depth of exploration and $\mathrm{EC}_{\mathrm{a}}$ (Callegary et al., 2007). For a combination of a vertical and horizontal dipole source-receiver with an offset $\rho$ over a multilayered earth, the electromagnetic forward model can be written as

$\mathrm{EC}_{\mathrm{a}}^{\mathrm{HCP}}(x, \rho)=\frac{-4 \rho}{\omega \mu_{0}} \operatorname{Im}\left[\int_{0}^{\infty} R_{0} J_{0}(\rho \lambda) \lambda^{2} \mathrm{~d} \lambda\right]$, 
$\mathrm{EC}_{\mathrm{a}}^{\mathrm{VCP}}(x, \rho)=\frac{-4}{\omega \mu_{0}} \operatorname{Im}\left[\int_{0}^{\infty} R_{0} J_{1}(\rho \lambda) \lambda \mathrm{d} \lambda\right]$.

In these expressions, $\mathrm{EC}_{\mathrm{a}}^{\mathrm{VCP}}$ and $\mathrm{EC}_{\mathrm{a}}^{\mathrm{HCP}}$ represent apparent electrical conductivity - measured in vertical and horizontal coplanar mode, $\mu_{0}$ represents permeability of the free space, $\lambda$ indicates the radial wave number, $J_{0}$ and $J_{1}$ correspond to the zero-order and first-order Bessel functions, $x$ is the depth of layer, $\omega$ is the angular frequency, and $\mathrm{Im}$ the quadrature component. The reflection factor $R_{0}$ is obtained recursively, starting from the lowest layer $N+1$, with $R_{N+1}=0$ :

$R_{n}\left(h_{n}, \sigma_{n}\right)=\frac{\frac{\Gamma_{n}-\Gamma_{n+1}}{\Gamma_{n}+\Gamma_{n+1}}+R_{n+1} \exp \left(-2 \Gamma_{n+1} h_{n+1}\right)}{1+\frac{\Gamma_{n}-\Gamma_{n+1}}{\Gamma_{n}+\Gamma_{n+1}} R_{n+1} \exp \left(-2 \Gamma_{n+1} h_{n+1}\right)}$,

$\Gamma_{n}=\sqrt{\lambda^{2}+\omega \mu_{0} j \sigma_{n}}$,

where $\sigma_{0}=0, h_{n}$ is the height, and $\sigma_{n}$ is the electrical conductivity for the $n$th layer. This is based on the assumption that each layer is uniform with infinite horizontal extent. EMI measurements were carried out under high induction number conditions $\left(\mathrm{EC}_{\mathrm{a}}>100 \mathrm{mS} \mathrm{m}^{-1}\right)$ utilizing the full solution of Maxwell's equation to model the forward EMI response.

\subsection{Bayesian inference}

Bayesian inference is used to express the uncertainties in the system parameters based on a suitable likelihood function and a prior. Given a set of unknown parameters, the so-called posterior distribution of the model parameters, which is the distribution of the parameters conditioned on available observations, is calculated as the product of the prior distribution and the likelihood function (Arulampalam et al., 2002; Sivia, 2006). Bayesian inversion has gained a lot of interest in recent years and has been applied in different applications, including climate, ocean and geophysical modeling (Malinverno, 2002; Zedler et al., 2012; Olson et al., 2012; Altaf et al., 2014; Sraj et al., 2014).

Suppose a set of observations $\left(\left\{y^{i}\right\}_{i=1}^{n}\right)$ is available and assume a certain model to predict the data. Let $\alpha$ be the set of unknown parameters in the model; then according to Bayes' rule,

$p\left(\alpha \mid\left\{y^{i}\right\}_{i=1}^{n}\right) \propto p\left(\left\{y^{i}\right\}_{i=1}^{n} \mid \alpha\right) p(\alpha)$,

where $p(\alpha)$ is the prior distribution of $\alpha$ that represents the a priori knowledge about $\alpha$ (i.e., before considering the data). The $p\left(\left\{y^{i}\right\}_{i=1}^{n} \mid \alpha\right)$ denotes the likelihood function: the probability of predicting the data given $\alpha$. The $p\left(\alpha \mid\left\{y^{i}\right\}_{i=1}^{n}\right)$ is the posterior probability: the probability of recovering $\alpha$ given the data $\left(\left\{y^{i}\right\}_{i=1}^{n}\right)$.

Let us consider the forward model $M$, for the evaluation of the observations $y$ as a function of the parameters such that

$y=M(\alpha)$.
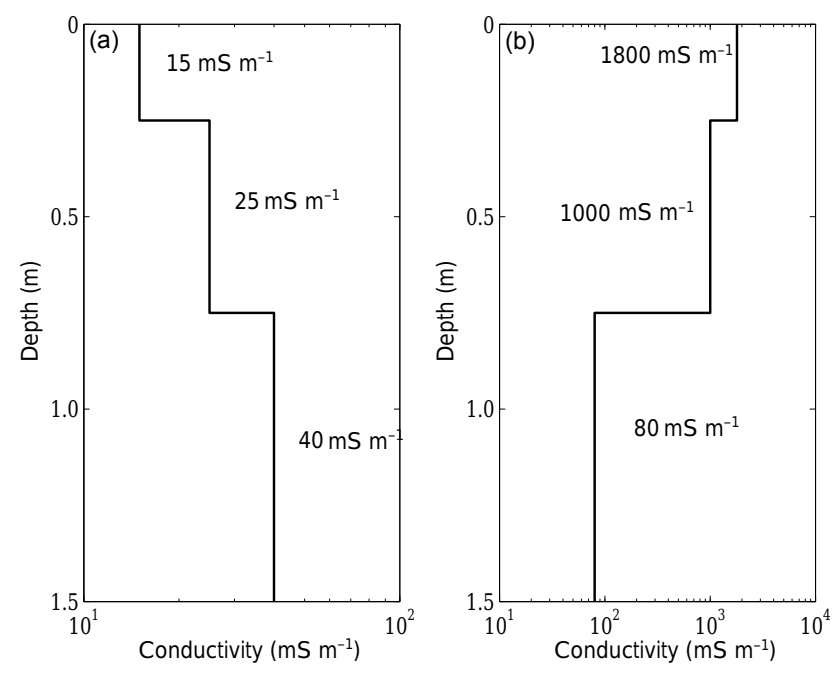

Figure 1. Three-layer synthetic earth model of electrical conductivity for (a) non-saline soil and (b) saline soil in the top horizon.

Let $\epsilon$ be a random variable representing the discrepancy between our model $M(\alpha)$ and the observations, which we refer to as the observational noise:

$\epsilon=y-M(\alpha)$.

Assuming the components of the observational noise to be independent and Gaussian of mean zero and variance $\sigma^{2}$, the likelihood function can then be decomposed as

$p\left(\left\{y^{i}\right\}_{i=1}^{n} \mid \alpha\right)=\prod_{i=1}^{n} \frac{1}{\sqrt{2 \pi \sigma^{2}}} \exp \left(-\frac{\left(y_{i}-M_{i}(\alpha)\right)^{2}}{2 \sigma^{2}}\right)$.

Here we consider $\sigma^{2}$ as an additional unknown (hyper) parameter and try to estimate its distribution as part of the inference process. The (joint) posterior distribution is then expressed as

$$
\begin{aligned}
& p\left(\alpha, \sigma^{2} \mid\left\{y^{i}\right\}_{i=1}^{n}\right) \propto \prod_{i=1}^{n} \frac{1}{\sqrt{2 \pi \sigma^{2}}} \\
& \quad \exp \left(-\frac{\left(y_{i}-M_{i}(\alpha)\right)^{2}}{2 \sigma^{2}}\right) p(\alpha) p\left(\sigma^{2}\right) .
\end{aligned}
$$

The choice of the prior is a key step in the inference process. Here, an informative uniform prior for all five (three conductivities and two thickness) parameters is considered, with $\alpha_{k}$ in the range $\left[\alpha_{k}^{\max } \alpha_{k}^{\min }\right]$; i.e.,

$p\left(\alpha_{k}\right)= \begin{cases}\frac{1}{\alpha_{k}^{\max }-\alpha_{k}^{\min }} & \text { for } \alpha_{k}^{\min }<\alpha_{k} \leq \alpha_{k}^{\max }, \\ 0 & \text { otherwise. }\end{cases}$

For the noise variance $\sigma^{2}$, we consider a Jeffreys prior (Sivia, 2006):

$p\left(\alpha_{k}\right)= \begin{cases}\frac{1}{\sigma^{2}} & \text { for } \sigma^{2}>0, \\ 0 & \text { otherwise. }\end{cases}$ 

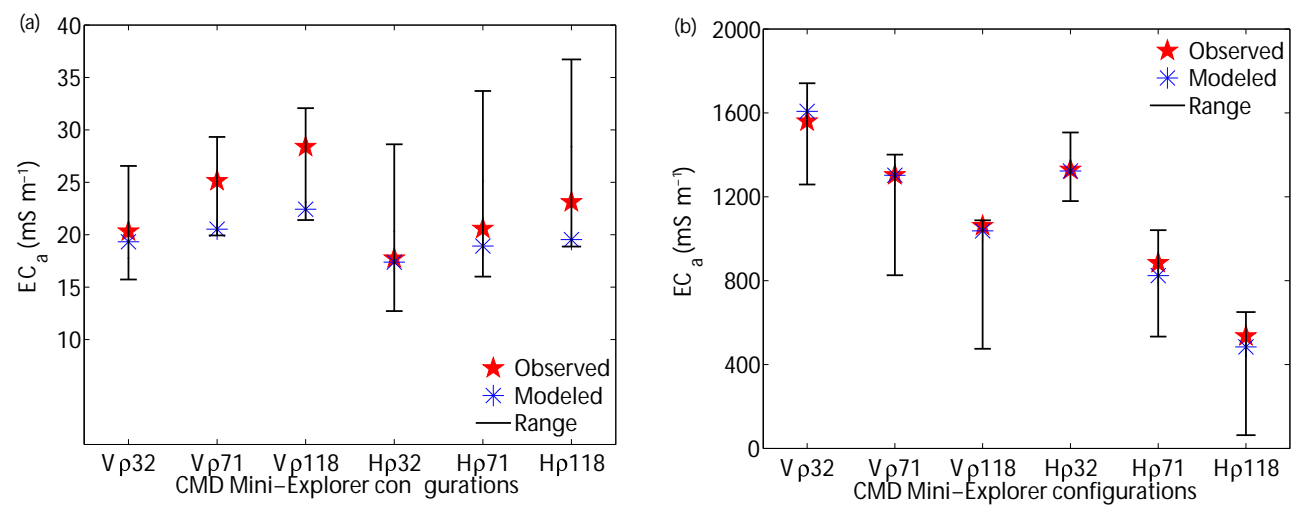

Figure 2. Observed electrical conductivity obtained from the forward response of the six different configuration of CMD Mini-Explorer (red star), estimated (modeled) earth electrical conductivity (blue asterisk) and the range of $\mathrm{EC}_{\mathrm{a}}$ simulated by MCMC for (a) non-saline and (b) saline soil scenarios.
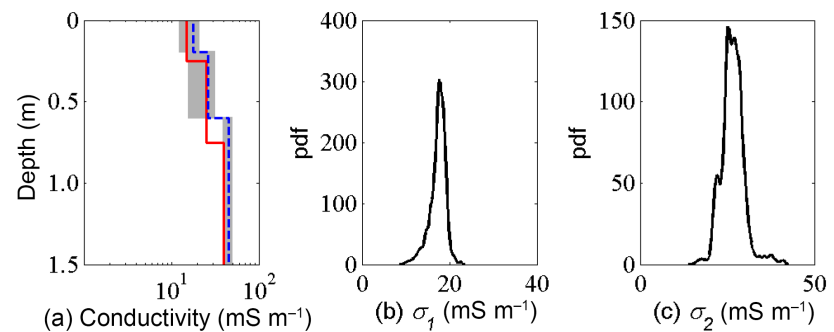

(a) Conductivity $\left(\mathrm{mS} \mathrm{m}^{-1}\right)$

(c) $\sigma_{2}\left(\mathrm{mS} \mathrm{m}^{-1}\right)$
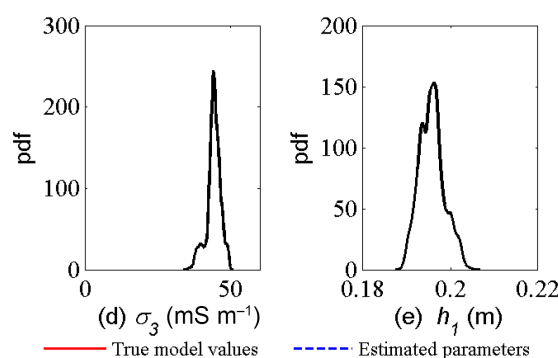

(e) $h_{1}(\mathrm{~m})$

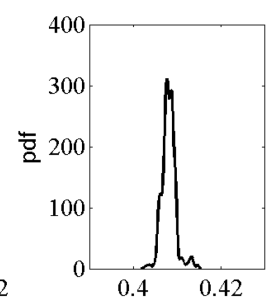

(f) $h_{2}(\mathrm{~m})$

KDF distribution $95 \%$ limi

Figure 3. Summary of the MCMC simulation for the synthetic three-layer earth model of non-saline soil. (a) True (red line) and estimated parameter (blue dashed line) for the vertical electrical conductivity profile, and the gray background with the $95 \%$ confidence interval of kernel distribution estimation (KDE). Panels (b-f) show the KDE of the marginalized posterior distributions for the three layer conductivities $\left(\sigma_{1}, \sigma_{2}\right.$, and $\left.\sigma_{3}\right)$ and the two layer thicknesses $\left(h_{1}\right.$ and $\left.h_{2}\right)$.

The most commonly used computational strategy to numerically solve a multidimensional parameters Bayesian inference problem is the Markov chain Monte Carlo (MCMC) method. We have applied an adaptive Metropolis MCMC algorithm to sample the posterior distribution, as described in details in Haario et al. (2001) and Roberts and Rosenthal (2009)

\subsection{Synthetic and field measurements}

Two sets of experimental setups were considered to test the MCMC approach and to evaluate the estimated model parameters and associated uncertainties using synthetic data for CMD Mini-Explorer configurations. Figure $1 \mathrm{a}$ and $\mathrm{b}$ show a three-layer earth model setups of low and high conductivity for non-saline soil and saline soil salinity, respectively. In both setups, thicknesses for the three-layer earth model were conceptualized by a plow horizon $(0.25 \mathrm{~m}$ thick), with an intermediate subsoil layer $(0.50 \mathrm{~m}$ thick) and underlying consolidated layer up to $1.5 \mathrm{~m}$ depth. The plowing horizon generally has less soil moisture as compared to the deeper horizon because of evaporation and infiltration processes. The scenario of non-saline soil therefore used a plowing horizon with low electrical conductivity of $15 \mathrm{mS} \mathrm{m}^{-1}$ as compared to the intermediate and consolidated soil layers (Fig. 1a). In the saline soil scenario, salt accumulates on the surface of soil due to evaporation of water. As a result, the electrical conductivity of plowing horizon is considered higher $1800 \mathrm{mS} \mathrm{m}^{-1}$ as compared to the deeper layers (Fig. 1b). In the agricultural field, the increase in the soil salinity is generally due to the use of poor water quality or the excessive use of fertilizers. The forward response of both scenarios was calculated in HCP and VCP via Eqs. (1) and (2), respectively, for EMI configuration setups using the characteristics of CMD MiniExplorer of three receiver coils respectively placed at 0.32 , 0.71 and $1.18 \mathrm{~m}$ distance from the receiver.

In both scenarios, six configurations, three for each HCP and VCP with different spacings, were taken as an output for forward models. Let $\alpha=\left(\sigma_{1}, \sigma_{2}, \sigma_{3}, h_{1}, h_{2}\right)^{T}$ be a vector of model parameters. $\sigma_{1}, \sigma_{2}$, and $\sigma_{3}$ are layer conductivities, and $h_{1}$ and $h_{2}$ thicknesses. Bayesian inference was used to estimate these five parameters that minimize the errors between observed and modeled HCP and VCP. An adaptive MCMC method was used to sample the posterior distributions and consequently update $\alpha$ distributions according to 


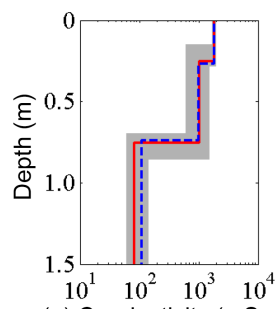

(a) Conductivity ( $\mathrm{mS} \mathrm{m}^{-1}$ )
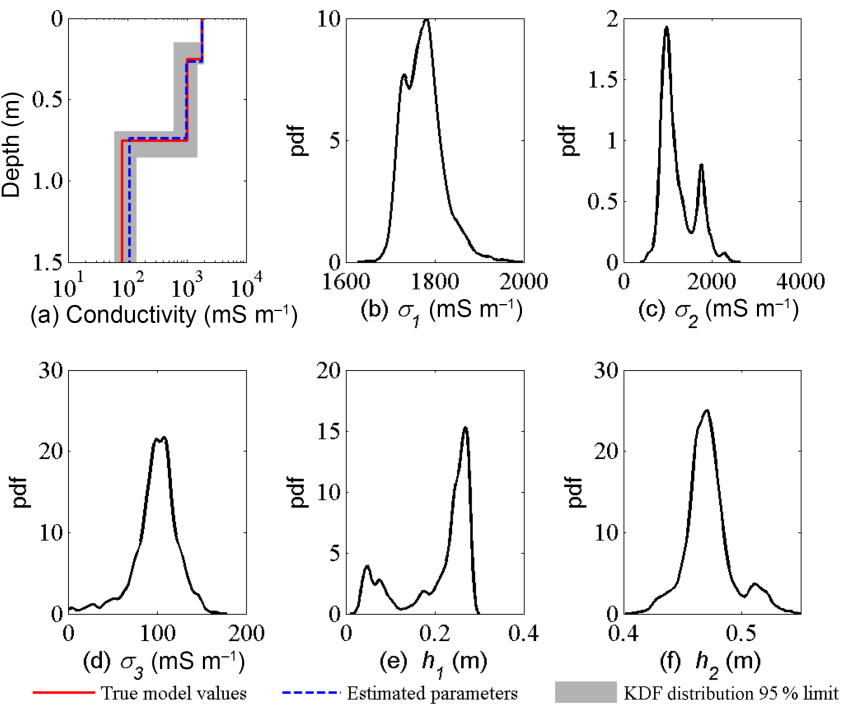

Figure 4. Summary of the MCMC simulation for the synthetic three-layer earth model of saline soil. (a) True (red line) and estimated parameter (blue dashed line) for the vertical electrical conductivity profile, and the gray background with the $95 \%$ confidence interval of kernel distribution estimation (KDE). Panels (b-f) show the $\mathrm{KDE}$ of the marginalized posterior distributions for the three layer conductivities $\left(\sigma_{1}, \sigma_{2}\right.$, and $\left.\sigma_{3}\right)$ and the two layer thicknesses $\left(h_{1}\right.$ and $\left.h_{2}\right)$.

the observed data. All the results presented below are based on $10^{4}$ MCMC samples. Parameter range for $h_{1}$ and $h_{2}$ was fixed between 0.05 and $0.6 \mathrm{~m}$ in each scenario. In the nonsaline scenario, parameter range for $\sigma_{1}, \sigma_{2}$, and $\sigma_{3}$ was considered between 5 and $100 \mathrm{mS} \mathrm{m}^{-1}$ and the saline soil scenario range was fixed between 5 and $3000 \mathrm{mS} \mathrm{m}^{-1}$. A uniform prior distribution function was considered in both scenarios.

Field measurements were also carried out in a farm, where Acacia trees were irrigated with saline groundwater. The farm is located at a distance of $6 \mathrm{~km}$ from the Red Sea coast at Al-Qadeimah, Makkah province, Saudi Arabia. EMI measurements were collected at an interval of $2 \mathrm{~m}$ over a $40 \mathrm{~m}$ long transect, along which three Acacia trees were irrigated using drip irrigation. At each location, EMI measurements using CMD Mini-Explorer system gives six different values of apparent electrical conductivity (using two coil orientations and three offsets); each responds to different depth ranges. Ten pits were dug along the same transect and in each pit the bulk electrical conductivity $\sigma_{\mathrm{b}}$ profile was measured at 15 locations within a depth range of $0.05-1.5 \mathrm{~m}$ via $5 \mathrm{TE}$ capacitance sensors (Decagon Devices, Pullman, USA). EMI and 5TE measurements were performed $8 \mathrm{~h}$ after the drip irrigation system was stopped, so that the soil moisture is not concentrated below the drippers and to give enough time to reduce the soil moisture impact due to evaporation, root water uptake, and infiltration (Jadoon et al., 2015).

\section{Results and discussion}

\subsection{Synthetic data}

Figure $2 \mathrm{a}$ and $\mathrm{b}$ depict the observed, estimated (modeled) and range of $\mathrm{EC}_{\mathrm{a}}$ as they result from the chain of MCMC simulation for six configurations of the synthetic case with saline and non-saline soil. The $x$ axis represents VCP and HCP with three coil spacing $(\rho 32, \rho 71, \rho 118)$. In a non-saline scenario, the layer electrical conductivity increases with depth (Fig. 1a), and this is reflected in the observed and modeled $\mathrm{EC}_{\mathrm{a}}$ in the VCP and HCP with increasing trend for larger spacing (Fig. 2a). The $\mathrm{EC}_{\mathrm{a}}$ value for the VCP and HCP with maximum spacing of $1.8 \mathrm{~m}$ between transmitter and receiver corresponds to deeper horizon; in the case of saline soil scenario the layer conductivity decreases (Fig. 1b) and as a result $\mathrm{EC}_{\mathrm{a}}$ values in VCP and HCP configuration exhibit a decreasing trend (Fig. 2b). The electromagnetic forward model is sensitive to high electrical conductive soil, so the modeled $\mathrm{EC}_{\mathrm{a}}$ values for the saline soil scenario match well with the observed as compared to the non-saline scenario. The mismatch between the observed and modeled $\mathrm{EC}_{\mathrm{a}}$ values for non-saline soil is due to the weak sensitivity of the forward electromagnetic model to the low electrical conductivity.

Figure $3 \mathrm{a}$ shows the true parameter values (red line) with the estimated parameters using MCMC (blue dashed line) for the non-saline soil scenario. The MCMC samples were used to obtain the marginalized posterior distributions based on kernel density estimation (KDE) (Parzen, 1962). The $95 \%$ confidence interval of the KDE for each parameter is shown by the shaded gray background (Fig. 3a). The resulting marginalized posterior probability density functions (PDFs) of the three conductivities and two thicknesses are shown in Fig. 3b-f. The estimated parameters (Fig. 3b-f) show a single peak, corresponding to the best parameter values. The electrical conductivities of the three model layers $\left(\sigma_{1}, \sigma_{2}\right.$, and $\left.\sigma_{3}\right)$ are reasonably well estimated as compared to the layer thicknesses. Different uniform prior distributions were also tested for the layer thicknesses, but the MCMC solution converged close to the prior instead of the true layer thicknesses. The topography of the objective function was too flat in this case to allow consequent changes in the direction of layer thicknesses. This suggests that the electromagnetic model is not sensitive to the layer thicknesses for the low-conductive soil layer.

Figure 4 illustrates the true and estimated depth profile of electrical conductivity for saline scenario, and the KDE of the marginalized posterior distributions for the three layer conductivities $\left(\sigma_{1}, \sigma_{2}\right.$, and $\left.\sigma_{3}\right)$ and the two layer thicknesses $\left(h_{1}\right.$ and $h_{2}$ ). The shaded gray background shows the $95 \%$ of the KDE for each parameter (Fig. 4a). The vertical electrical conductivity profile is well recovered by MCMC. The electrical conductivity of the top two layers are well estimated as compared to the consolidated layer with low electrical conductivity. Furthermore, for the six tested configurations of 

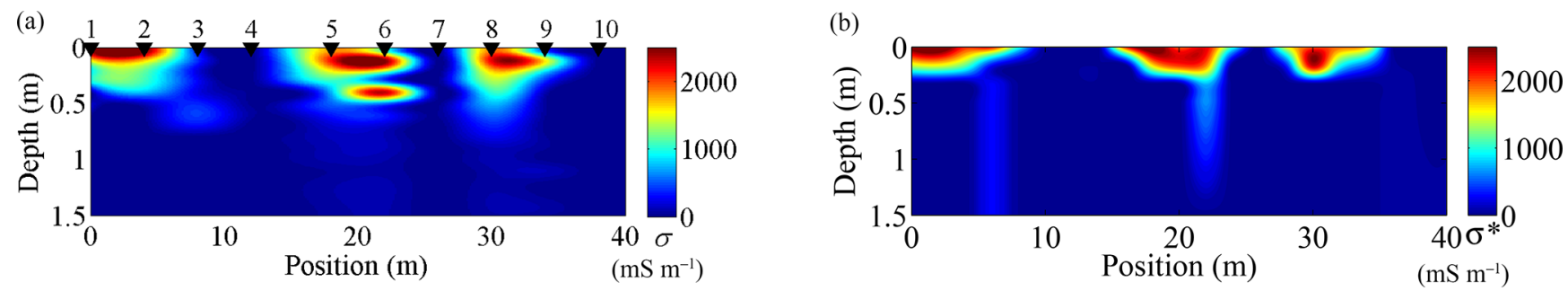

Figure 5. (a) Electrical conductivity $\left(\mathrm{mS} \mathrm{m}^{-1}\right)$ measured by the $5 \mathrm{TE}$ capacitance sensors from 10 soil pits along transect and the location of the soil pits is indicated by black triangles (Jadoon et al., 2015); (b) the soil electrical conductivity obtained by using Markov chain Monte Carlo simulation for multi-configuration electromagnetic induction measurements.
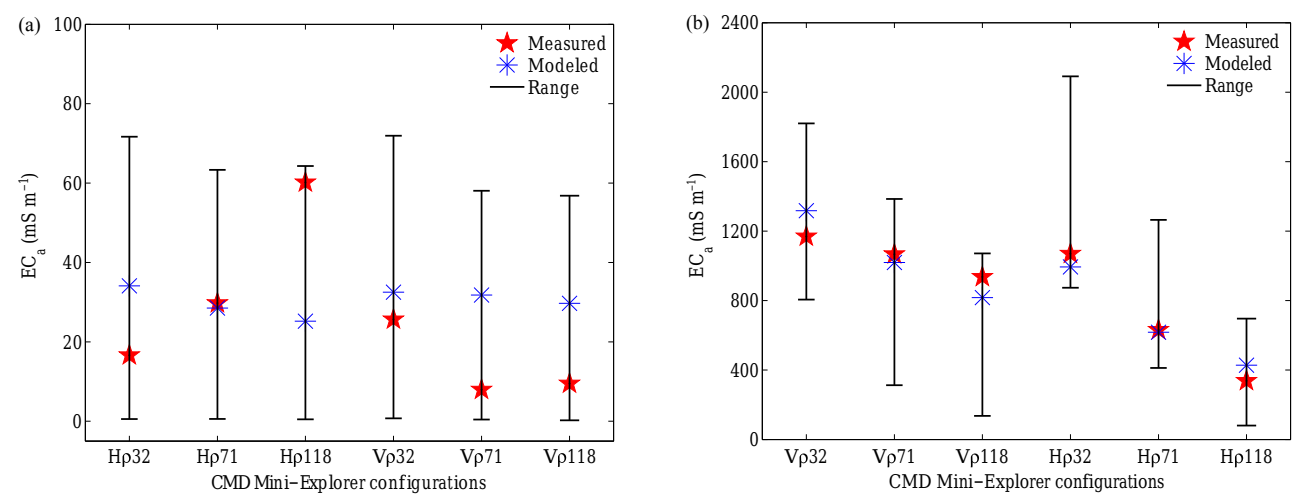

Figure 6. Measured six different configuration of CMD Mini-Explorer (red star), estimated (modeled) earth electrical conductivity (blue asterisk) and the range of $\mathrm{EC}_{\mathrm{a}}$ simulated by $\mathrm{MCMC}$ for (a) non-saline soil at pit 4 and (b) saline soil at pit 9 location.

CMD Mini-Explorer, the HCP and VCP configuration with spacing $1.18 \mathrm{~m}$ are mostly sensitive to the consolidated layer while the remaining four configurations are more sensitive to the upper horizon. A large range of the parameter space was explored by MCMC (Fig. 4b-e), illustrating the sensitivity of the electromagnetic model to the considered parameters.

\subsection{Experimental data}

Measurements were carried out in a farm, where acacia trees were irrigated with saline groundwater. The farm is located at a distance of $6 \mathrm{~km}$ from the Red Sea coast at Al-Qadeimah, Makkah province, Saudi Arabia. EMI measurements were collected at an intervals over a $40 \mathrm{~m}$ long transect, along which three acacia trees were irrigated using drip irrigation. At each location, EMI measurements using the CMD MiniExplorer system provides six different values of apparent electrical conductivity (using two coil orientations and three offsets); each responds to different depth ranges. Ten pits were dug along the same transect and in each pit the vertical $\sigma_{\mathrm{b}}$ profile was measured at 15 locations within a depth range of $0.05-1.5 \mathrm{~m}$ via 5TE capacitance sensors (Decagon Devices, Pullman, USA). 5TE and EMI measurements were carried out on the same day $8 \mathrm{~h}$ after the drip irrigation system was stopped, so that the soil moisture concentration below the drippers is avoided, and enough time is given for the reduction of soil moisture impact due to root water uptake, evaporation and infiltration (Jadoon et al., 2015).

Figure 5 shows the soil electrical conductivity measured in ten pits along a transect and the modeled soil electrical conductivity as estimated by the MCMC using the multi-configuration EM induction measurements. Pit locations along the transect are indicated by black triangle and cubic interpolation of 150 5TE sensor measurements were used to construct the two-dimensional profile of measured soil electrical conductivity $\sigma$ (Fig. 5a). The groundwater used to irrigate the acacia trees has an electrical conductivity of $4200 \mathrm{mS} \mathrm{m}^{-1}$. The three patterns of high electrical conductivity is due to the infiltration front and soil salinity near the three acacia trees. In total, 21 multi-configuration EMI measurements were performed along a transect and calibrated with in situ measurements collected using capacitance sensors (Jadoon et al., 2015). The three-layer earth model was considered for Bayesian inference of the five parameters $\left(\sigma_{1}, \sigma_{2}, \sigma_{3}, h_{1}, h_{2}\right)$ and their uncertainty based on the 15000 MCMC samples. For all MCMC simulations, the parameter search space was set relatively large, with the range of low and high values of electrical conductivity of soil; $0<\sigma_{1}<3000 \mathrm{mS} \mathrm{m}^{-1}, 0<\sigma_{2}<3000 \mathrm{mS} \mathrm{m}^{-1}, 0<\sigma_{3}<$ $3000 \mathrm{mS} \mathrm{m}^{-1}, 0.05<h_{1}<0.6 \mathrm{~m}$, and $0.05<h_{1}<0.6 \mathrm{~m}$. In the depth section of soil electrical conductivity resulting from the EMI MCMC simulations, the effect of infiltration pat- 


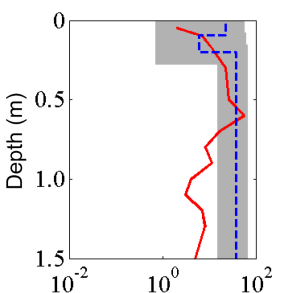

(a) Conductivity $\left(\mathrm{mS} \mathrm{m}^{-1}\right)$

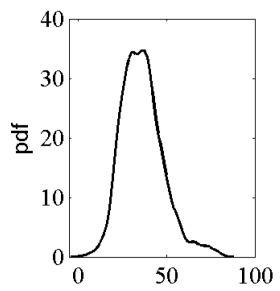

(d) $\sigma_{3}\left(\mathrm{mS} \mathrm{m}^{-1}\right)$

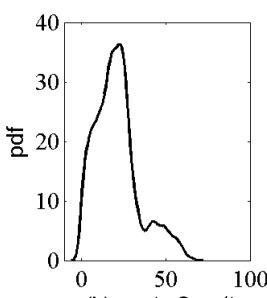

(b) $\sigma_{1}\left(\mathrm{mS} \mathrm{m}^{-1}\right)$

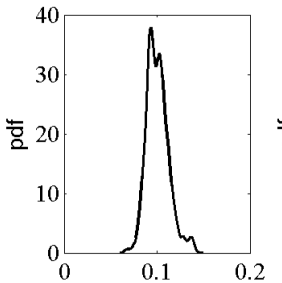

(e) $h_{1}(\mathrm{~m})$

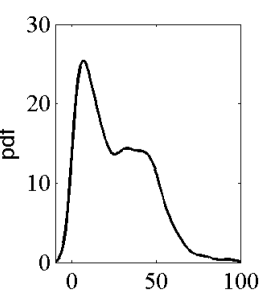

(c) $\sigma_{2}\left(\mathrm{mS} \mathrm{m}^{-1}\right)$

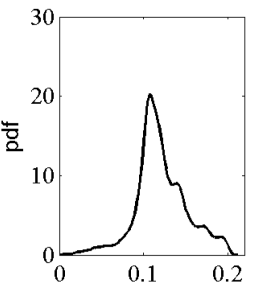

(f) $h_{2}(\mathrm{~m})$

KDF distribution $95 \%$ limit

Figure 7. Summary of the MCMC simulation for three-layer earth model by considering CMD Mini-Explorer measurement over a non-saline soil. (a) True (red line) and estimated parameter (blue dashed line) for the vertical electrical conductivity profile, and the gray background with the $95 \%$ confidence interval of kernel distribution estimation (KDE). (b-f) The KDE of the marginalized posterior distributions for the three layer conductivities $\left(\sigma_{1}, \sigma_{2}\right.$, and $\left.\sigma_{3}\right)$ and two layer thicknesses $\left(h_{1}\right.$ and $\left.h_{2}\right)$.

terns and the soil salinity due to the drip irrigation near the three acacia trees is clear (Fig. 5b). The estimated soil electrical conductivity values by MCMC are in a good agreement with the sensor measurements performed in pits (Fig. 5a).

Figure $6 \mathrm{a}$ and $\mathrm{b}$ show the measured, estimated (modeled) and range of $\mathrm{EC}_{\mathrm{a}}$ as they result from the $\mathrm{MCMC}$ chain for the six multi-configurations of CMD Mini-Explorer for saline and non-saline soil. Three coil spacings for each VCP and $\mathrm{HCP}$ are represented on the $x$ axis. EMI measurement is shown for non-saline and saline soil at locations 4 and 9 of the pit (Fig. 5a), respectively. The soil was completely dry for non-saline soil as no irrigation was applied, whereas in the case of saline soil the moisture in the soil varied between 0.005 and 0.19 at the time of EMI and sensor measurements. In non-saline soil, the measured six $\mathrm{EC}_{\mathrm{a}}$ values range between 5 and $60 \mathrm{mS} \mathrm{m}^{-1}$ and the modeled $\mathrm{EC}_{\mathrm{a}}$ between 23 and $38 \mathrm{mS} \mathrm{m}^{-1}$ (Fig. 6a). The range of $\mathrm{EC}_{\mathrm{a}}$ estimated from the last $10000 \mathrm{MCMC}$ samples is in the range of $0-75 \mathrm{mS} \mathrm{m}^{-1}$. As observed in the synthetic non-saline soil scenario, the electromagnetic forward model was not sensitive to the low electrical conductive soil. Similarly, the fit between the measured and modeled $\mathrm{EC}_{\mathrm{a}}$ is not in good agreement with the real measurements (Fig. 6a). Furthermore, the misfit may be due to the large search parameter space in the MCMC simulations. In the case of saline soil, the electrical conductivity of the top $50 \mathrm{~cm}$ soil is high due to the saline infiltration and soil salinity. This effect can be seen in the decreasing trend of the measured $\mathrm{EC}_{\mathrm{a}}$ for the VCP and HCP

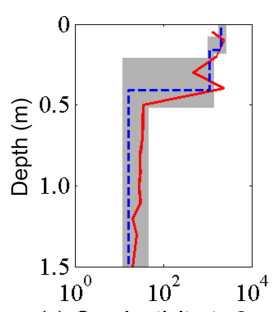

(a) Conductivity $\left(\mathrm{mS} \mathrm{m}^{-1}\right)$

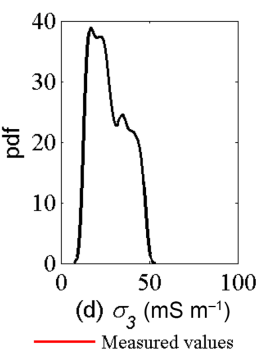

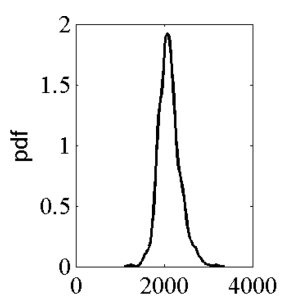

(b) $\sigma_{1}\left(\mathrm{mS} \mathrm{m}^{-1}\right)$

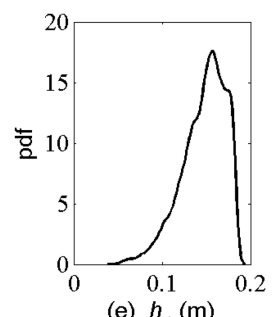

(e) $h_{1}(\mathrm{~m})$

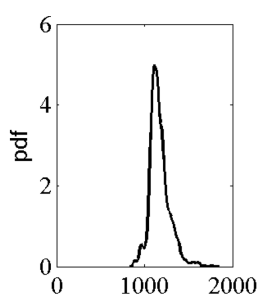

(c) $\sigma_{2}\left(\mathrm{mS} \mathrm{m}^{-1}\right)$

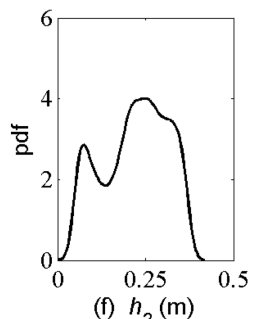

Figure 8. Summary of the MCMC simulation for three-layer earth model by considering CMD Mini-Explorer measurement over a saline soil. (a) True (red line) and estimated parameter (blue dashed line) for the vertical electrical conductivity profile, and the gray background with the $95 \%$ confidence interval of kernel distribution estimation (KDE). (b-f) The KDE of the marginalized posterior distributions for the three layer conductivities $\left(\sigma_{1}, \sigma_{2}\right.$, and $\left.\sigma_{3}\right)$ and two layer thicknesses $\left(h_{1}\right.$ and $\left.h_{2}\right)$.

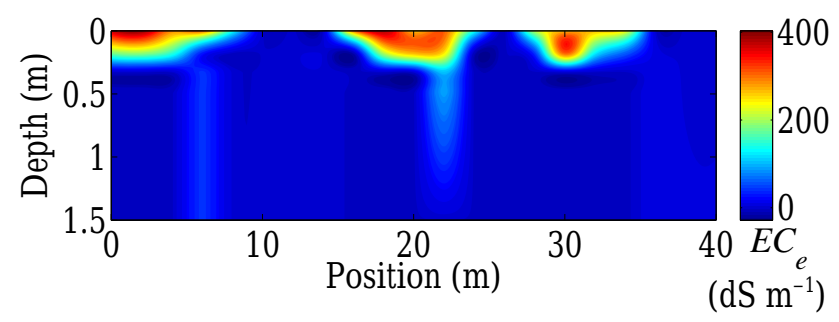

Figure 9. Spatial distribution of soil salinity $\left(\mathrm{EC}_{\mathrm{e}}\right)$ obtained using Bayesian inversion of multi-configuration EMI measurements along a transect.

measurements with larger coil spacing (Fig. 6b). The measured and modeled $\mathrm{EC}_{\mathrm{a}}$ are in good agrement and this is due to the high sensitivity of the electromagnetic forward model to high electrical conductive soil.

Figure 7 plots the vertical profile of electrical conductivity for non-saline soil as measured by capacitance sensors (red line), the value of the MCMC estimated parameters (blue dashed line), and the KDE of the marginalized posterior distributions for the three layer conductivities and the two layer thicknesses. The CMD Mini-Explorer measurements at pit 4 for non-saline soil were used for the analysis. In Fig. 7a the measured vertical profile of soil electrical conductivity falls within the shaded area in the top 95\% KDE distribution limit $0-0.7 \mathrm{~m}$ depth and below this depth the modeled soil electrical conductivity is overestimated. The mismatch between the measured and modeled $\mathrm{EC}_{\mathrm{a}}$ for the maximum coil 
separation $H \rho 118$ and $V \rho 118$ is behind the overestimation of the soil electrical conductivity. The marginalized posterior PDFs of the three conductivities and two thicknesses are shown in Fig. 7b-f. The PDFs of the parameters (Fig. 7b-f) exhibit a single peak, corresponding to the best parameters. The peak of the $\sigma_{3}$ is flat between 30 and $38 \mathrm{mS} \mathrm{m}^{-1}$ and it seems that the topography of the objective function does not change within this range of conductivity in each iteration of the MCMC simulation.

Figure 8 plots the vertical profile of electrical conductivity for the saline soil measured by capacitance sensors (red line), the value of the MCMC estimated parameters (blue dashed line), and the $\mathrm{KDE}$ of the marginalized posterior distributions for the three layer conductivities and the two layer thicknesses. CMD Mini-Explorer measurements at pit 9 for saline soil was used for the analysis. The shaded area in Fig. 8a indicates the $95 \%$ KDE distribution limits. The whole measured vertical profile of soil electrical conductivity falls within the shaded area, suggesting that the electrical conductivity is well estimated. The marginalized posterior PDFs of the three conductivities and two thicknesses, as shown in Fig. 8b-f, exhibit a single peak for all parameters except the layer thickness $h_{2}$ which is flat, suggesting that the data were not informative to refine our prior knowledge about $h_{2}$. The posterior PDFs of the first two conductivities $\left(\sigma_{1}\right.$ and $\left.\sigma_{2}\right)$ and layer thickness $h_{1}$ exhibit a clear Gaussian shape with an obvious maximum a posteriori (MAP) estimate. For the conductivity parameter $\sigma_{3}$, we notice a posterior with a well-defined peak, but no standard PDF shape.

Figure 9 shows the spatial distribution of the soil salinity as estimated from EMI measurement using MCMC. Soil salinity $\mathrm{EC}_{\mathrm{e}}$ is related to bulk electrical conductivity $\sigma_{\mathrm{b}}$ via a linear relationship $\left(\mathrm{EC}_{\mathrm{e}}=13.74 \sigma_{\mathrm{b}}+0.001\right)$ established by Jadoon et al. (2015) for the same site. Infiltration front and high soil salinity range between 0.01 and $0.5 \mathrm{~m}$ at three locations where acacia trees are irrigated with brackish water. The results show that the Bayesian inversion of multiconfiguration EMI measurements successfully estimates the soil salinity caused by the brackish water infiltration. In the field, acacia tree roots concentrated in the top $70 \mathrm{~cm}$ of soil and the low soil salinity below $30 \mathrm{~cm}$ shows that acacia are capable of extracting salt solutions and reducing subsoil salinity.

\section{Conclusion}

In this paper, an adaptive Bayesian MCMC algorithm has been implemented for the model assessment and uncertainty analysis of multi-orientation and multi-offset EMI measurements. The algorithm has been tested for CMD MiniExplorer with both synthetic and field measurements conducted in an agriculture field over a non-saline and saline soil. Using Bayesian inference, marginalized posterior PDFs were computed for three subsurface electrical conductivities $\left(\sigma_{1}, \sigma_{2}\right.$, and $\left.\sigma_{3}\right)$ and two layer thicknesses $\left(h_{1}\right.$ and $\left.h_{2}\right)$ using MCMC. Such analysis helps to provide insight about parameter estimates and uncertainties.

The experimental results showed that the MCMC simulations can improve the reliability of the electromagnetic forward model to estimate the subsurface electrical conductivity profiles. Analysis shows that the electromagnetic forward model is less sensitive to the non-saline soil as compared to the saline soil. The proposed approach is flexible and can be implemented for various low-frequency ground-based EMI systems and can provide subsurface electrical conductivity distribution and uncertainty of model parameters. Future research will focus on implementing the Bayesian inference approach on time-lapse EMI measurements in different agricultural fields to monitor the soil dynamics, and estimate the model parameters and their uncertainties.

Data availability. The data were acquired at a privately owned farm in Saudi Arabia and are not available to the public.

Competing interests. The authors declare that they have no conflict of interest.

Acknowledgements. This research was funded by the Water Desalination and Reuse Center, King Abdullah University of Science and Technology (KAUST), Saudi Arabia.

Edited by: Monica Riva

Reviewed by: one anonymous referee

\section{References}

Altaf, M. U., Butler, T., Mayo, T., Luo, X., Dawson, C., Heemink, A. W., and Hoteit, I.: A Comparison of Ensemble Kalman Filters for Storm Surge Assimilation, Mon. Weather Rev., 142, 28992914, 2014.

Anderson, W. L.: Numerical integration of related Hankel transforms of orders 0 and by adaptive digital filtering, Geophysics, 44, 1287-1305, 1979.

Arulampalam, M. S., Maskell, S., Gordon, N., and Clapp, T.: A tutorial on particle filters for online nonlinear/non-Gaussian Bayesian tracking, IEEE T. Signal Proces., 50, 174-188, 2002.

Callegary, J. B., Ferre, T. P. A., and Groom, R. W.: Vertical spatial sensitivity and exploration depth of low-induction-number electromagnetic-induction instruments, Vadose Zone J., 6, 158167, 2007.

Cook, P. G. and Walker, G. R.: Depth profiles of electricalconductivity from linear-combinations of electromagnetic induction measurements, Soil Sci. Soc. Am. J., 56, 1015-1022, 1992.

Corwin, D. L.: Past, present, and future trends of soil electrical conductivity measurement using geophysical methods, in: Handbook of Agricultural Geophysics, edited by: Allred, B., Daniels, 
J. J., and Ehsani, M. R., CRC Press, Taylor and Francis Group, Boca Raton, Folrida, 17-44, 2008.

Corwin, D. L. and Lesch, S. M.: Apparent soil electrical conductivity measurements in agriculture, Comput. Electron. Agr., 46, 11-43, 2005a.

Corwin, D. L. and Lesch, S. M.: Characterizing soil spatial variability with apparent soil electrical conductivity: I. Survey protocols, Comput. Electron. Agr., 46, 103-134, 2005 b.

Ershadi, A., McCabe, M. F., Evans, J. P., Chaney, N. W., and Wood, E. F.: Multi-site evaluation of terrestrial evaporation models using FLUXNET data, Agr. Forest Meteorol., 187, 46-61, 2014.

Haario, H., Saksman, E., and Tamminen, J.: An adaptive Metropolis algorithm, Bernoulli, 7, 223-242, 2001.

Hendrickx, J. M. H., Borchers, B., Corwin, D. L., Lesch, S. M., Hilgendorf, A. C., and Schlue, J.: Inversion of soil conductivity profiles from electromagnetic induction measurements: Theory and experimental verification, Soil Sci. Soc. Am. J., 66, 673-685, 2002.

Huang, H. P. and Won, I. J.: Real-time resistivity sounding using a hand-held broadband electromagnetic sensor, Geophysics, 68, 1224-1231, 2003.

Jadoon, K. Z., Moghadas, D., Jadoon, A., Missimer, T. M., AlMashharawi, S. K., and McCabe, M. F.: Estimation of soil salinity in a drip irrigation system by using joint inversion of multicoil electromagnetic induction measurements, Water Resour. Res., 51, 3490-3504, 2015.

Keller, G. and Frischknecht, F.: Electrical methods in geophysical prospecting. International Series of Monographs in Electromagnetic Waves 10, Pergamon Press, Oxford, New York, 1966.

Li, H. Y., Shi, Z., Webster, R., and Triantafilis, J.: Mapping the three-dimensional variation of soil salinity in a rice-paddy soil, Geoderma, 195, 31-41, 2013.

Malinverno, A.: Parsimonious Bayesian Markov chain Monte Carlo inversion in a nonlinear geophysical problem, Geophys. J. Int., 151, 675-688, 2002.

McNeill, J. D.: Electromagnetic terrain conductivity measurement at low induction numbers, Tech note TN-6, Geonics Ltd., Mississauga, ON, Canada, 10, 1319-1330, 1980.

Mester, A., van der Kruk, J., Zimmermann, E., and Vereecken, H.: Quantitative Two-Layer Conductivity Inversion of MultiConfiguration Electromagnetic Induction Measurements, Vadose Zone J., 10, 1319-1330, 2011.

Minsley, B. J.: A trans-dimensional Bayesian Markov chain Monte Carlo algorithm for model assessment using frequency-domain electromagnetic data, Geophys. J. Int., 187, 252-272, 2011.

Olson, R., Sriver, R., Goes, M., Urban, N. M., Matthews, H. D., Haran, M., and Keller, K.: A climate sensitivity estimate using Bayesian fusion of instrumental observations and an Earth System model, J. Geophys. Res.-Atmos., 117, D04103, https://doi.org/10.1029/2011JD016620, 2012.
Parzen, E.: On Estimation of a Probability Density Function and Mode, Ann. Math. Stat., 33, 1065-1076, 1962.

Rhoades, J. D., Raats, P. A. C., and Prather, R. J.: Effects of Liquidphase Electrical Conductivity, Water Content, and Surface Conductivity on Bulk Soil Electrical Conductivity, Soil Sci. Soc. Am. J., 40, 651-655, 1976.

Roberts, G. O. and Rosenthal, J. S.: Examples of Adaptive MCMC, J. Comput. Graph. Stat., 18, 349-367, 2009.

Robinson, D. A., Lebron, I., Kocar, B., Phan, K., Sampson, M., Crook, N., and Fendorf, S.: Time-lapse geophysical imaging of soil moisture dynamics in tropical deltaic soils: An aid to interpreting hydrological and geochemical processes, Water Resour. Res., 45, W00D32, https://doi.org/10.1029/2008WR006984, 2009.

Santos, F. A. M., Triantafilis, J., Taylor, R. S., Holladay, S., and Bruzgulis, K. E.: Inversion of Conductivity Profiles from EM Using Full Solution and a 1-D Laterally Constrained Algorithm, J. Environ. Eng. Geoph., 15, 163-174, 2010.

Scharnagl, B., Vrugt, J. A., Vereecken, H., and Herbst, M.: Inverse modelling of in situ soil water dynamics: investigating the effect of different prior distributions of the soil hydraulic parameters, Hydrol. Earth Syst. Sci., 15, 3043-3059, https://doi.org/10.5194/hess-15-3043-2011, 2011.

Sivia, D. S.: Data Analysis: A Bayesian Tutorial, Oxford Science Publications, Oxford, UK, 2006.

Sraj, I., Mandli, K. T., Knio, O. M., Dawson, C. N., and Hoteit, I.: Uncertainty quantification and inference of Manning's friction coefficients using DART buoy data during the Tohoku tsunami, Ocean Model., 83, 82-97, 2014.

Sudduth, K. A., Kitchen, N. R., Bollero, G. A., Bullock, D. G., and Wiebold, W. J.: Comparison of electromagnetic induction and direct sensing of soil electrical conductivity, Agron. J., 95, 472482, 2003.

Triantafilis, J. and Monteiro Santos, F. A.: Electromagnetic conductivity imaging (EMCI) of soil using a DUALEM-421 and inversion modelling software (EM4Soil), Geoderma, 211, 28-38, 2013.

von Hebel, C., Rudolph, S., Mester, A., Huisman, J. A., Kumbhar, P., Vereecken, H., and van der Kruk, J.: Three-dimensional imaging of subsurface structural patterns using quantitative largescale multiconfiguration electromagnetic induction data, Water Resour. Res. 50, 2732-2748, 2014.

Zedler, S. E., Kanschat, G., Korty, R., and Hoteit, I.: A new approach for the determination of the drag coefficient from the upper ocean response to a tropical cyclone: a feasibility study, J. Oceanogr., 68, 227-241, 2012. 